\title{
Synthesis of Tetragonal Barium Titanate in the Concentrated Light Energy Field
}

\author{
M.S. Payzullakhanov \\ Institute of Material-sciences SPA "Physics-Sun” AS RUz 100084 Tashkent, Bodomzor Road str., 2-B \\ *Corresponding Author: fayz@bk.ru
}

Copyright (C) 2013 Horizon Research Publishing All rights reserved.

\begin{abstract}
Barium titanate ceramic samples were synthesized from mixture of $\mathrm{TiO}_{2}+\mathrm{BaCO}_{3}$ under influence of concentrated light flux of $200 \mathrm{Wt} / \mathrm{cm}^{2}$ at $2000^{\circ} \mathrm{C}$ followed by quenching (A-type) and by the ceramic technology at $1350^{\circ} \mathrm{C}$ followed by cooling at the rate of $100{ }^{0} \mathrm{C} / \mathrm{min}$ (B-type). The both types of samples had tetragonal symmetry lattice with the parameters $\mathrm{a}=0.399 \mathrm{~nm}$ and $\mathrm{c}=0.403 \mathrm{~nm}$, however their tetragonality degree $\delta=\mathrm{c} / \mathrm{a}-1$ slightly differed (0.01 and 0.007 for A- and B-type, respectively). Dielectric permittivity value was 3800 for A-type and 3400 for B-type; bending deformation began at $66 \mathrm{MPa}$ for A-type and $49 \mathrm{MPa}$ for B-type.
\end{abstract}

Keywords Syntheses, Light Energy, Material

\section{Introduction}

Ferroelectric and piezoelectric materials as functional ceramics have structure sensitive properties and are capable of energy transformation. Barium titanate is known to have four crystal modifications. High temperature modification is characterized by cubic structure of perovskite type and does not have ferroelectric properties. Other three low temperature modifications of barium titanate have a lower symmetry and advanced ferroelectric properties in wide temperature range [1-3]. It is quite difficult to grow crystals of barium titanate large enough for cutting plates suitable for applications. Fortunalety, it is possible to implement ceramic technology to obtain ferroelectric barium titanate polycrystal.

Spontaneous polarization intrinsic for ferroelectrics relates with domains of single oriented polarization. Domain structure depends on crystal symmetry, nature and distribution of defects in crystal lattices. The type and number of defects depend on the parameters of synthesis technology. Study of material synthesis in the field of concentrated light has shown that the rate of physical-chemical processes of structure formation and destruction increases under effect of solar radiation. It is possible to fix high temperature phases of definite nano-state with various defectiveness degree under short effect of concentrated light flux of high density (up to $700 \mathrm{Wt} / \mathrm{cm}^{2}$ ) resulting in melting, followed by superfast quenching (to $10^{5}$ $\mathrm{grad} / \mathrm{s})$. Varying the technological parameters of light impact allows one to control the defectiveness of synthesized material. However, radiation and thermal physical and chemical mechanisms of structurization and destruction at influence of concentrated light flux on several materials have not been understood yet.

This work is aimed at studying of synthesis of ferroelectric materials based on barium titanate in the concentrated light flux of high density and also their dielectric and mechanical properties.

\section{Materials and Techniques}

Chemically pure grade powders of $\mathrm{TiO}_{2}$ and $\mathrm{BaCO}_{3}$ were mixed in accordance with the stoichiometrical composition of barium titanate (in mass ratio) $\mathrm{BaCO}_{3}: \mathrm{TiO}_{2}=1.9: 1$ and PVA binder. Sample-pellets with the diameter of $18 \mathrm{~mm}$ and thickness of $2 \mathrm{~mm}$ were pressed at $500 \mathrm{kN}$ and fixed in the focal plane of the optical furnace of URAN type, the scheme of which is shown in Fig. 1.

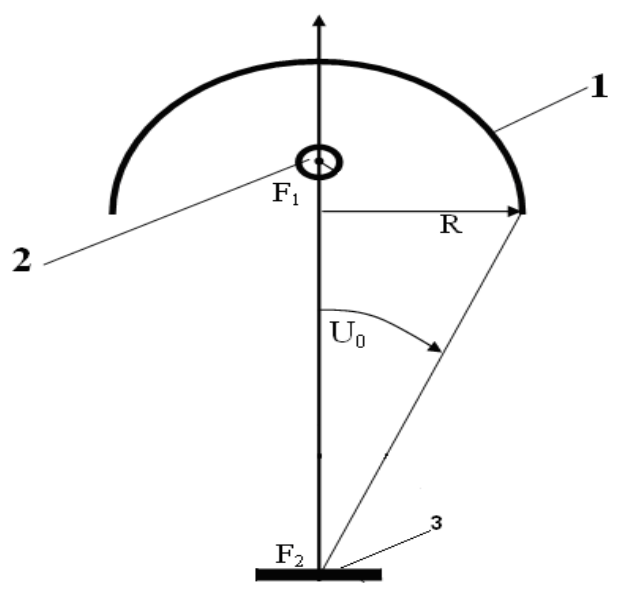

Figure 1. Scheme of optical system "Uran"; 1 - reflector, 2 - xenon lamp, 3 - optical focus.

The source of radiation is placed in the near focus of 
ellipsoid (F1), and the receiver is in the far focus (F2). Xenon lamp of $10 \mathrm{~kW}$ is used as a radiation source emitting in visible and near infrared ranges with the maximal intensity in the wavelength interval of 1.0-2.0 micron. The efficiency (ration of radiation flux to electric power) of such lamps is about $60 \%$. The density in the focus was measured by the detector F0A0 13-07, the temperature was monitored by optical pyrometer of PROMIN type by GOST 8335-81.

The value of light flux power corresponding to the necessary temperature was determined by the formulae $\mathrm{Q}=\left(\mathrm{a}_{\mathrm{s}} / \varepsilon\right) \sigma \mathrm{T}^{4}$, where $\mathrm{Q}-$ power of radiation, $\mathrm{a}_{\mathrm{s}}$ and $\varepsilon-$ coefficients of absorption and emission, respectively, $\sigma$ Boltzmann constant $5.67 \times 10^{-8} \mathrm{~W} \cdot \mathrm{m}^{-2} \cdot \mathrm{K}^{-4}, \mathrm{~T}-$ temperature, $\mathrm{K}$.

Calculated temperature at the light flux density of 200 $\mathrm{W} / \mathrm{cm}^{2}$ is $2270 \mathrm{~K}$ that exceeds the melting point of $\mathrm{BaTiO}_{3}$ $(1900 \mathrm{~K})$. Molten samples were quenched in water at the rate of $10^{4} \mathrm{grad} / \mathrm{sec}$, that allowed us to fix high temperature metastable states of $\mathrm{BaTiO}_{3}$.

Phase composition, structure parameters of the samples were determined at the installation DRON-3 with $\mathrm{Cu}-\mathrm{K}_{\alpha}$-radiation $(\lambda=0.15418 \mathrm{~nm})$ selected by the monochromator with $\mathrm{Ni}-$ filter. The crystal lattice parameters were determined by using the extrapolation -Raylegh function [4].

The quenched molten samples were milled and pressed into pellet-samples as mentioned above. The samples were sintered at the temperature of $1350^{\circ} \mathrm{C}$ during 2 hours followed by cooling at $100 \mathrm{grad} / \mathrm{min}$ (samples of A-type). To evaluate the effect of solar light, the reference samples were sintered from the same mixture in the electric furnace at the same temperature and cooled at the same rate (samples of B-type).

The densities of the sintered samples of A- and B types determined by gygroscopic weighing were 4.21 and 4.12 $\mathrm{g} / \mathrm{cm}^{3}$, respectively.

Dielectric parameters were measured with IPD-1 at the frequencies of $0.3,1.0,3.0,10,20,30 \mathrm{kHz}$. Special Ni contacts were vacuum deposited on the sample pellets.

The dielectric permittivity of the samples was calculated by the formulae:

$$
\varepsilon=\frac{h}{\varepsilon_{0} S} C_{x}
$$

where $h$ - sample thickness, $\mathrm{m} ; S$ - sample area, $\mathrm{m}^{2} ; \varepsilon_{0}-$ dielectric constant $8.85 \cdot 10^{-12} \mathrm{~F} / \mathrm{m} ; C_{\mathrm{x}}-$ measured value, $\mathrm{pF}$.

Crystallite size of the powder was estimated from the peaks of X-ray reflections by the Scherrer formulae

$$
d=\frac{0.9 \lambda}{F W H M \cos \vartheta}
$$

Where $d$ - crystallite size $(\mathrm{nm}), \lambda$ - wavelength of monochromatized $X$-ray radiation $(\lambda=0.154056 \mathrm{~nm}$ for $\mathrm{CuK} \alpha$ emission), FWHM - the peak width at the semiheight of diffraction reflection (rad), $\theta$ - diffraction angle (deg).

The degree of crystallinity (DC) was calculated on the area under the diffraction reflection peaks :

$$
D C=\frac{A_{\text {crystallinity }}}{\left(A_{\text {crystallinity }}+A_{\text {amorphous }}\right)} * 100
$$

where $\mathrm{A}_{\text {crystallinity }}$ and $\mathrm{A}_{\text {amorphous }}$ are the areas under the peaks of crystalline and amorphous reflections.

\section{Results and Discussion}

Figure 2 depicts X-ray diffraction spectrum of A-type sample. There are well distinct diffraction reflections of various intensities. Analysis has shown that such a picture is well described by diffraction of X-rays from tetragonal lattice $(\mathrm{RF}=4.6 \%)$ with the lattice parameters $a=0.399 \mathrm{~nm}$, $c=0.403 \mathrm{~nm}$. The factor of tetragonality $\delta=c / a-1=0.010$ evidents on deformation of the lattice.

Figure 3 shows X-ray reflections 200 and 002 for the samples of A- (a) and B- (b) types.

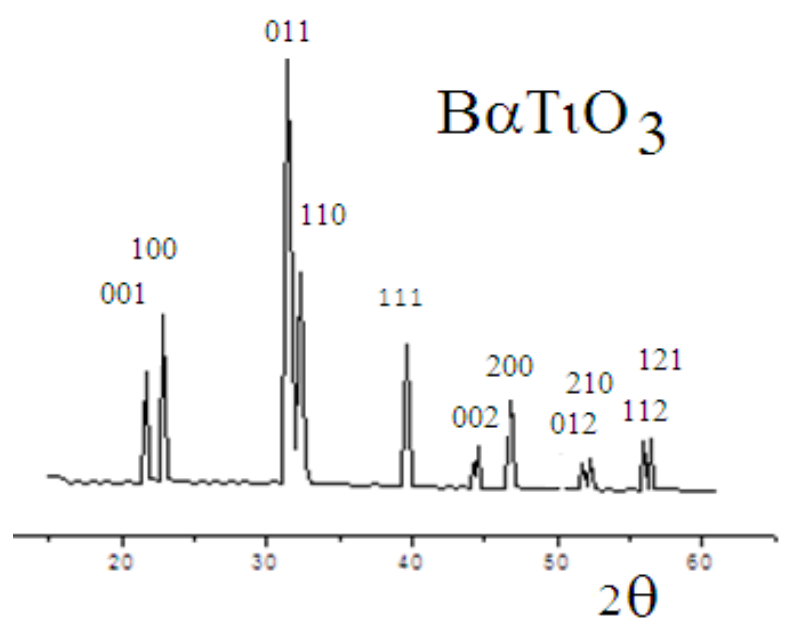

Figure 2. X-ray diffraction spectrum of the sample sintered at $1350^{\circ} \mathrm{C}$.

$a$

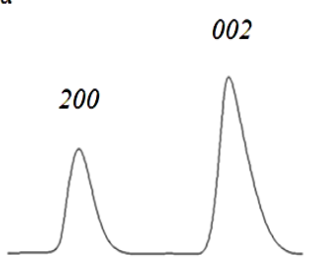

$\tilde{\sigma}$

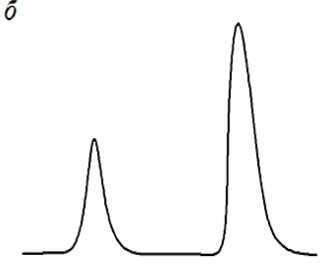

Figure 3. X-ray diffractions 200 and 002 for samples A- (a) and B- (b) types.

Given the values of $\mathrm{FWHM}_{002}=7 \times 10^{-3} \mathrm{rad}$ and 
$\mathrm{FWHM}_{200}=6 \times 10^{-3} \mathrm{rad}$, the crystallite size calculated by Scherrer formulae was $d \sim 30 \mathrm{~nm}$.

For B-type samples the lattice parameters $a=0.398 \mathrm{~nm}$, $c=0.401 \mathrm{~nm}$ differed from those for A-type samples. Calculation shows, that the tetragonality degree 0.007 for B-type samples is $25 \%$ less than that of A-type. The crystallite size is $d \sim 80 \mathrm{~nm}$.

It is known that both a small size of particles or crystallites and high defectiveness degree cause broadening of X-ray diffraction profiles.

Figure 4 demonstrates electron microscopy photos of barium titanate samples synthesized with the use of concentrated light flux (A-type) and solid phase reaction techniques (B-type).

Sample of A-type has the structure of close packed polyhedral crystals of various shapes with sizes from 40 to $140 \mathrm{~nm}$. Sample of B-type consists of polyhedral crystallites of various shapes separate and close packed with the sizes from 100 to $1250 \mathrm{~nm}$. They have also prevailing orientation in c-axis.

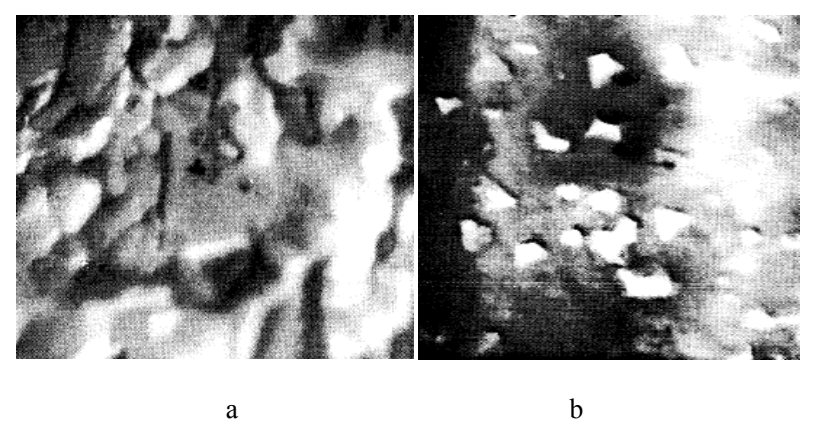

Figure 4. Electron microscopy photos of barium titanate samples synthesized with the use of concentrated light flux (A-type) and solid phase reaction techniques (B-type).

It is seen that polycrystalline barium titanate is ceramics with chaotically distributed separate microcrystallites and interlayers of glasslike phase. The amount of glassy phase varies from 1 mass. $\%$ to a few tens percents depending on the sintering temperature and degree. At high sintering temperatures $\left(>1350^{\circ} \mathrm{C}\right)$ the amount of glass phase makes $1-$ 2 mass.\%. Such microstructure provides the ceramics with high mechanical strength.

Measurements of bending deformation of barium titanate samples were done at the installation FR-100. It was found that destruction of the samples having the shape of a bar with sizes $5 \times 5 \times 10 \mathrm{~mm}$ begins at $66 \mathrm{MPa}$ (A-type) and $54 \mathrm{MPa}$ (B-type), which is quite high value of the bending strength $P_{b}$.

Ceramic barium titanate being piezoelectrics should have a large value of dielectric permittivity: 3800 (A-type) and 3400 (B-type) at room temperature. These values exceed very much those given in $[5,6]$. The tangent of dielectric losses was small: $\operatorname{tg} \delta \approx 0.031$ (A-type) and $\approx 0.037$ (B-type).

\section{Conclusions}

Thus, tetragonal barium titanate with $\delta=(c / a-1)=0.01$ synthesized by melting of $\mathrm{TiO}_{2}+\mathrm{BaCO}_{3}$ mixture under effect of concentrated light flux of $200 \mathrm{~W} / \mathrm{cm}^{2}$ has tiny crystallites with sizes from 40 to $140 \mathrm{~nm}$ and an advanced dielectric constant $\varepsilon=3800$ and also the bending strength $\mathrm{P}_{\mathrm{b}}$. Reference samples synthesized by the ceramic technology have $40 \%$ less $\delta$ and $12 \%$ lower $\varepsilon$ and also $18 \%$ less $\mathrm{P}_{\mathrm{b}}$.

\section{REFERENCES}

[1] M. M. Vijatović, J. D. Bobić, B. D. Stojanovic. History and Challenges of Barium Titanate. Journal Science of Sintering, 2008, vol. 40, pp.155-165.

[2] J. H. Lee, C. W. Won, T. S. Kim, H. S. Kim. Characteristics of $\mathrm{BaTiO} 3$ powders synthesized by hydrothermal process. Journal of Materials Science, 2000, vol. 35, pp. 4271-4274.

[3] G. Busch Early history of ferroelectricty. Ferroelectrics. 1987. Vol. 74, pp.267-284

[4] Mirkin L.I. X-Ray diffraction analysis. Moscow: MIR, 1961, $237 \mathrm{p}$.

[5] B.M. Gorlov, E.V. Kotenok, S.N. Mahno, V.V. Sidorchuk, S.V. Halameida, V.A. Zazhigalov. Structure, optical and dielectric properties of barium titanate nanoparticles obtained by mechanochemical method. Journal of Technical Physics, 2011, vol.81, pp.87-94.

[6] G. Wang, X. Chen, Y. Duan, S. Liu. Journal of Alloys and Compounds, 2008, vol. 454, pp. 340-346. 